\title{
Weighted stochastic field exponent Sobolev spaces and nonlinear degenerated elliptic problem with nonstandard growth
}

\author{
Ismail Aydın' (D), Cihan Unal*2 (D) \\ ${ }^{1}$ Department of Mathematics, Sinop University, 57000 Sinop, Turkey \\ ${ }^{2}$ Assessment, Selection and Placement of Center, 06800 Ankara, Turkey
}

\begin{abstract}
In this study, we consider weighted stochastic field exponent function spaces $L_{\vartheta}^{p(., .)}(D \times \Omega)$ and $W_{\vartheta}^{k, p(. .)}(D \times \Omega)$. Also, we study some basic properties and embeddings of these spaces. Finally, we present an application for defined spaces to the stochastic partial differential equations with stochastic field growth.
\end{abstract}

Mathematics Subject Classification (2010). 46E35, 43A15, 60H15

Keywords. quasilinear elliptic equation, weighted stochastic field exponent Sobolev spaces, pseudo-monotone operator, compact embedding theorem

\section{Introduction}

Nonlinear partial differential equations arise in chemical and biological problems, in formulating fundamental laws of nature, in different areas of physics, applied mathematics and engineering (such as solid mechanics, fluid dynamics, acoustics, nonlinear optics, plasma physics, quantum eld theory) and numerous applications. To study these equations is a very difficult task because there are no general methods to solve such equations. Moreover, the existence and the uniqueness of the solutions are fundamental, hard-toprove questions for any nonlinear equation with given boundary conditions.

In various applications (such as elasticity, non-Newtonian fluids and electrorheological fluids, see [13]), it can be seen the boundary value obstacle problems for elliptic equations. Many of these type equations have been investigated for constant exponents of nonlinearity but it seems to be more realistic to assume the variable exponent, see [4].

Harjulehto et. al [8] investigate an overview of applications to differential equations with non-standard growth. Also, Aoyama [1] considered the some properties of variable exponent Lebesgue spaces on a probability space. In 2014, Tian et. al [15] introduced stochastic field exponent function spaces $L^{p(. .)}(D \times \Omega)$ and $W^{k, p(. .)}(D \times \Omega)$. They gave also an application to the stochastic partial differential equations with stochastic field growth in these spaces. Moreover, Lahmi et. al [10] proved the existence of solutions

\footnotetext{
*Corresponding Author.

Email addresses: iaydin@sinop.edu.tr (I. Aydın), cihanunal88@gmail.com (C. Unal)

Received: 08.05.2019; Accepted: 10.10.2019
} 
for the nonlinear $p($.$) -degenerate problems involving nonlinear operators. This study is a$ generalization of $[10,14,15]$.

In this paper, we define weighted stochastic field exponent function spaces $L_{\vartheta}^{p(., .)}(D \times \Omega)$ and $W_{\vartheta}^{k, p(., .)}(D \times \Omega)$, and discuss some basic properties of these spaces. Finally, we discuss the existence and uniqueness of the weak solution for the nonlinear degenerated weighted $p(.,$.$) elliptic problem$

$$
\left\{\begin{array}{cc}
-\operatorname{div} A(x, t, u, \nabla u)+A_{0}(x, t, u, \nabla u)=f(x, t), & (x, t) \in D \times \Omega \\
u=0, & (x, t) \in \partial D \times \Omega
\end{array}\right.
$$

where $A(x, t, s, \xi)$ and $A_{0}(x, t, s, \xi)$ are Carathéodory functions, which are measurable stochastic fields on $D \times \Omega$ and continuous for $s$ and $\xi$ under some conditions. Moreover, $f(x, t)$ is an integrable stochastic field on $D \times \Omega$. The measurable stochastic field $p(.,$.$) :$ $D \times \Omega \longrightarrow[1, \infty)$ satisfies $1<p^{-} \leq p(.,.) \leq p^{+}<\infty$.

It is known that pseudo-monotone operators have been many applications in nonlinear elliptic equations. As an example, we can refer Browder [2]. Here, the author investigated a class of pseudo-monotone operators and applied it to a kind of boundary value problems for nonlinear elliptic equations.

By the theory of pseudo-monotone operators, our aim is to show the compactness techniques and the existence of a least weak solution of (1.1). The special case of the equation of (1.1) is the following equation

$$
\left\{\begin{array}{cc}
-\operatorname{div}\left(\vartheta(x, t)|\nabla u|^{p(x, t)-2} \nabla u\right)+\vartheta(x, t) g(u)|\nabla u|^{p(x, t)-1}=f(x, t), & (x, t) \in D \times \Omega \\
u=0, & (x, t) \in \partial D \times \Omega
\end{array} .\right.
$$

Now, suppose that $\lambda$ is a product measure on $D \times \Omega$ and $u(x, t)$ is a Lebesgue measurable stochastic field on $D \times \Omega$, where $D$ is a bounded open subset of $\mathbb{R}^{d}(d>1)$, and $(\Omega, \Lambda, P)$ is a complete probability space.

\section{Weighted stochastic field exponent Lebesgue and Sobolev spaces}

Definition 2.1. We denote the family of all measurable functions $p(.,):. D \times \Omega \longrightarrow[1, \infty)$ (called a stochastic field exponent). In this paper, the function $p(.,$.$) always denotes a$ stochastic field exponent. Moreover, we put

$$
p^{-}=\operatorname{essinf}_{(x, t) \in D \times \Omega} p(x, t), \quad p^{+}=\operatorname{essup}_{(x, t) \in D \times \Omega} p(x, t) .
$$

A positive, measurable and locally integrable function $\vartheta$ defined on $D \times \Omega$ is called a weight function. Now, we introduce the integrability conditions used on the weighted variable Lebesgue and Sobolev spaces

$$
\begin{array}{ll}
\left(H_{1}\right): & \vartheta \in L_{l o c}^{1}(D \times \Omega) \text { and } \vartheta^{-\frac{1}{p(., .)-1}} \in L_{l o c}^{1}(D \times \Omega) \\
\left(H_{2}\right): & \vartheta^{-s(., .)} \in L^{1}(D \times \Omega),
\end{array}
$$

with $s(.,.) \in\left(\frac{d}{p(. .)}, \infty\right) \cap\left[\frac{1}{p(. .)-1}, \infty\right)$. The weighted modular function $\rho_{p(. .,), \vartheta}$ on $D \times \Omega$ is defined by

$$
\rho_{p(., .), \vartheta}(u)=E\left(\int_{D}|u(x, t)|^{p(x, t)} \vartheta(x, t) d x\right)=\int_{D \times \Omega}|u(x, t)|^{p(x, t)} \vartheta(x, t) d \lambda,
$$

where $d \lambda=d \lambda(x, t)=d x d t$. 
The spaces $L_{\vartheta}^{p(., .)}(D \times \Omega)$ consist of all measurable stochastic fields (functions) $u$ on $D \times \Omega$ such that $\int_{D \times \Omega}|u(x, t)|^{p(x, t)} \vartheta(x, t) d \lambda<\infty$ and endowed with the Luxemburg norm

$$
\|u\|_{p(., .), \vartheta}=\inf \left\{\tau>0: \int_{D \times \Omega}\left|\frac{u(x, t)}{\tau}\right|^{p(x, t)} d x d t \leq 1\right\} .
$$

It is well known that $u \in L_{\vartheta}^{p(. .)}(D \times \Omega)$ if and only if the norm $\|u\|_{p(. .,), \vartheta}=\left\|u \vartheta^{\frac{1}{p(., .)}}\right\|_{p(. .,)}$ is finite. Moreover, it is clear that, if the inequality $0<C \leq \vartheta$ is satisfied, then $L_{\vartheta}^{p(. .)}(D \times \Omega) \hookrightarrow L^{p(., .)}(D \times \Omega)$. In this study, we assume that $1<p^{-} \leq p(.,.) \leq p^{+}<\infty$ and $\left(H_{1}\right),\left(H_{2}\right)$. Moreover, we will use the abbreviations and symbols; a.e., $\longrightarrow$ and $\rightarrow$ for almost everywhere, strong convergence and weak convergence, respectively.

It can be seen that the space $L_{\vartheta}^{p(. .)}(D \times \Omega)$ is uniformly convex, so it is reflexive, see [5]. Moreover, we denote by $L_{\vartheta *}^{q(. .)}(D \times \Omega)$ as the dual space of $L_{\vartheta}^{p(., .)}(D \times \Omega)$ where $\frac{1}{p(., .)}+\frac{1}{q(., .)}=1$ and $\vartheta^{*}=\vartheta^{1-q(., .)}$.

Proposition 2.2 (Hölder-type inequality, see [5]). Let $p(.) \in L_{+}^{\infty}(\Omega)$. The conjugate space to $L^{p(.)}(\Omega)$ is $L^{p^{\prime}(.)}(\Omega)$, where $\frac{1}{p(x)}+\frac{1}{p^{\prime}(x)}=1$ for almost every $x \in \Omega$. Moreover, the following inequality hold

$$
\left|\int_{\Omega} j(x) h(x) d x\right| \leq\left(1+\frac{1}{p^{-}}-\frac{1}{p^{+}}\right)\|j\|_{p(.)}\|h\|_{p^{\prime}(.)}
$$

for all $j \in L^{p(.)}(\Omega)$ and $h \in L^{p^{\prime}(.)}(\Omega)$.

Now, we present the relationships between $\|\cdot\|_{p(.,), \vartheta}$ and $\rho_{p(., .), \vartheta}$ as follows. The proof is clear.

Proposition 2.3 (see [15]). If $u \in L_{\vartheta}^{p(. .)}(D \times \Omega)$, then we have

(i) $\|u\|_{p(., .), \vartheta}^{p^{-}} \leq \rho_{p(., .), \vartheta}(u) \leq\|u\|_{p(., .), \vartheta}^{p^{+}}$with $\|u\|_{p(., .), \vartheta} \geq 1$.

(ii) $\|u\|_{p(., .), \vartheta}^{p^{+}} \leq \rho_{p(. .,), \vartheta}(u) \leq\|u\|_{p(., .), \vartheta}^{p^{-}}$with $\|u\|_{p(., .), \vartheta} \leq 1$.

Theorem 2.4. Let $\frac{1}{p(. .)}+\frac{1}{q(. .)}=1$ and $\vartheta^{*}=\vartheta^{1-q(.)}$. Then we have

$$
E\left(\int_{D}|f(x, t) g(x, t)| d x\right) \leq C\|f\|_{p(., .), \vartheta}\|g\|_{q(., .), \vartheta *}
$$

for every $f \in L_{\vartheta}^{p(. . .)}(D \times \Omega)$ and $g \in L_{\vartheta^{*}}^{q(. .)}(D \times \Omega)$ with the constant $C$ depends on $p(.,$.$) .$

Proof. If we consider the Hölder inequality, then we get

$$
\begin{aligned}
E\left(\int_{D}|f(x, t) g(x, t)| d x\right) & =E\left(\int_{D}|f(x, t) g(x, t)|(\vartheta(x, t))^{\frac{1}{p(x, t)}-\frac{1}{p(x, t)}} d x\right) \\
& \leq C\left\|f \vartheta^{\frac{1}{p(. .)}}\right\|_{p(. . .)}\left\|g \vartheta^{-\frac{1}{p(. .)}}\right\|_{q(. .)}
\end{aligned}
$$

for some $C>0$.

Theorem 2.5 (see $[14,15])$. The space $L_{\vartheta}^{p(., .)}(D \times \Omega)$ is a reflexive Banach space with respect to norm $\|\cdot\|_{p(., .), \vartheta}$. 
Proposition 2.6. The space $L_{\vartheta}^{p(. . .)}(D \times \Omega)$ is continuously embedded in $L_{l o c}^{1}(D \times \Omega)$. This means that every function in $L_{\vartheta}^{p(. . .)}(D \times \Omega)$ has distributional (weak) derivative.

Proof. Suppose that $u \in L_{\vartheta}^{p(. .)}(D \times \Omega)$ and let $K=K_{1} \times K_{2} \subset D \times \Omega$ be a compact set. By the Hölder inequality, there is an $A_{K}>0$ such that

$$
\begin{aligned}
\rho_{p(., .), K}(u) & =E\left(\int_{K_{1}}|u(x, t)| \vartheta^{\frac{1}{p(x, t)}} \vartheta^{-\frac{1}{p(x . t)}} d x\right) \\
& \leq A_{K}\left\|u \vartheta^{\frac{1}{p(. .)}}\right\|_{p(.,), K}\left\|\vartheta^{-\frac{1}{p(. .)}}\right\|_{q(., .), K}
\end{aligned}
$$

where $\frac{1}{p(. .)}+\frac{1}{q(., .)}=1$. It is obvious that $\left\|\vartheta^{-\frac{1}{p(. .)}}\right\|_{q(., .), K}<\infty$ if and only if $\rho_{q(., .), K}\left(\vartheta^{-\frac{1}{p(. .)}}\right)<\infty$. Since $\vartheta^{-\frac{1}{p(., .)-1}} \in L_{l o c}^{1}(D \times \Omega)$, we have

$$
\rho_{q(., .), K}\left(\vartheta^{-\frac{1}{p(. .)}}\right)=E\left(\int_{K_{1}} \vartheta^{-\frac{q(x, t)}{p(x, t)}} d x\right)=\int_{K} \vartheta^{-\frac{1}{p(x, t)-1}} d \lambda=B_{K}<\infty .
$$

If we consider (2.1) and (2.2), then we have the desired result.

Remark 2.7. If $\vartheta^{-\frac{1}{p(., .)-1}} \notin L_{l o c}^{1}(D \times \Omega)$, then the space $L_{\vartheta}^{p(., .)}(D \times \Omega)$ might not be continuously embedded in $L_{l o c}^{1}(D \times \Omega)$.

Theorem 2.8. Let $u \in L_{\vartheta}^{p(. .)}(D \times \Omega)$ and $u_{n} \in L_{\vartheta}^{p(., .)}(D \times \Omega)$ with $\left\|u_{n}\right\|_{p(., .), \vartheta} \leq C$ for some $C>0$. If $u_{n} \longrightarrow$ a.e. in $D \times \Omega$, then $u_{n} \rightarrow u$ in $L_{\vartheta}^{p(. . .)}(D \times \Omega)$.

Proof. Since the space $L_{\vartheta}^{p(. .)}(D \times \Omega)$ is reflexive, we only need to see that

$$
E\left(\int_{D} u_{n} g d x\right) \rightarrow E\left(\int_{D} u g d x\right)
$$

for every $g \in L_{\vartheta^{*}}^{q(. .)}(D \times \Omega)$ where $\frac{1}{p(., .)}+\frac{1}{q(., .)}=1$ and $\vartheta^{*}=\vartheta^{1-q(., .)}$. It is well known that $\left\|u_{n}\right\|_{p(.,), \vartheta} \leq C$ if and only if $\rho_{p(., .), \vartheta}\left(\frac{u_{n}}{C}\right) \leq 1$ for every $n \in \mathbb{N}$. This follows by the Fatou's Lemma that

$$
E\left(\int_{D}\left|\frac{u}{C}\right|^{p(x, t)} \vartheta(x, t) d x\right) \leq \liminf _{n \longrightarrow \infty} E\left(\int_{D}\left|\frac{u_{n}}{C}\right|^{p(x, t)} \vartheta(x, t) d x\right) \leq 1 .
$$

Thus, we get $\|u\|_{p(., .),} \leq C$. By the absolute continuity of the Lebesgue integral, we have

$$
\lim _{\operatorname{meas}(K) \rightarrow 0} \int_{D \times \Omega}\left|g \chi_{K}\right|^{q(x, t)} \vartheta^{*}(x, t) d \lambda=0
$$

where $g \in L_{\vartheta^{*}}^{q(.,)}(D \times \Omega)$ and $K \subset D \times \Omega$. This yields that $\lim _{\operatorname{meas}(K) \longrightarrow 0}\left\|g \chi_{K}\right\|_{q(., .), \vartheta^{*}}=0$, and there is a $\delta>0$ such that

$$
\left\|g \chi_{K}\right\|_{q(., .), \vartheta^{*}}<\frac{\varepsilon}{4 C}\left(1+\frac{1}{p^{-}}-\frac{1}{p^{+}}\right)^{-1}
$$

for meas $(K)<\delta$. If we consider the Egorov theorem, then there is a set $L \subset D \times \Omega$ such that $u_{n} \longrightarrow u$ uniformly on $L$ with meas $((D \times \Omega)-L)<\delta$. Moreover, if we choose $n_{0}$ 
such that $n \geq n_{0}$, then we have

$$
\max _{(x, t) \in L}\left|u_{n}-u\right|\|g\|_{q(., .), \vartheta^{*}}\left\|\chi_{L}\right\|_{p(., .), \vartheta}\left(1+\frac{1}{p^{-}}-\frac{1}{p^{+}}\right)<\frac{\varepsilon}{2} .
$$

Let us denote $K=(D \times \Omega)-L$. By (2.3) and (2.4), we have

$$
\begin{aligned}
& \left|E\left(\int_{D} u_{n} g d x\right)-E\left(\int_{D} u g d x\right)\right| \\
& \quad \leq \int_{L}\left|u_{n}-u\right||g| d \lambda+\int_{K}\left|u_{n}-u\right||g| d \lambda \\
& \quad \leq \max _{(x, t) \in L}\left|u_{n}-u\right| E\left(\int_{D}\left|g \chi_{L}\right| d x\right)+E\left(\int_{D}\left|u_{n}-u\right|\left|g \chi_{K}\right| d x\right) \\
& \quad \leq \max _{(x, t) \in L}\left|u_{n}-u\right|\|g\|_{q(. . .), \vartheta^{*}}\left\|\chi_{L}\right\|_{p(. . .), \vartheta}\left(1+\frac{1}{p^{-}}-\frac{1}{p^{+}}\right) \\
& \quad+\left\|u_{n}-u\right\|_{p(. . .), \vartheta}\left\|g \chi_{K}\right\|_{q(. . .), \vartheta^{*}}\left(1+\frac{1}{p^{-}}-\frac{1}{p^{+}}\right) \\
& <\varepsilon .
\end{aligned}
$$

That is the desired result.

Definition 2.9. The weighted stochastic field variable Sobolev spaces $W_{\vartheta}^{k, p(.,)}(D \times \Omega)$ defined by

$$
W_{\vartheta}^{k, p(., .)}(D \times \Omega)=\left\{u \in L_{\vartheta}^{p(., .)}(D \times \Omega): D^{\alpha} u \in L_{\vartheta}^{p(. .)}(D \times \Omega), 0 \leq|\alpha| \leq k\right\}
$$

equipped with the norm

$$
\|u\|_{W_{\vartheta}^{k, p(.,)}(D \times \Omega)}=\sum_{0 \leq|\alpha| \leq k}\left\|D^{\alpha} u\right\|_{p(., .), \vartheta},
$$

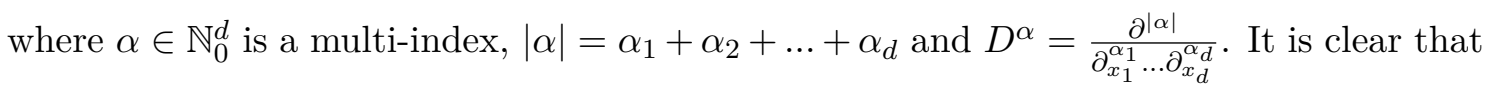
the space $\left(W_{\vartheta}^{k, p(., .)}(D \times \Omega),\|\cdot\|_{W_{\vartheta}^{k, p(. .)}(D \times \Omega)}\right)$ is a reflexive Banach space by $[15$, Theorem $2.5]$.

Moreover, the space $W_{\vartheta}^{1, p(., .)}(D \times \Omega)$ is defined by

$$
W_{\vartheta}^{1, p(., .)}(D \times \Omega)=\left\{u \in L_{\vartheta}^{p(. .)}(D \times \Omega):|\nabla u| \in L_{\vartheta}^{p(. .)}(D \times \Omega)\right\}
$$

with the norm $\|u\|_{W_{\vartheta}^{1, p(., .)}(D \times \Omega)}=\|u\|_{p(., .),}+\|\nabla u\|_{p(.,), \vartheta}$.

The space $W_{0, \vartheta}^{1, p(. .,)^{\prime}}(D \times \Omega)$ is the closure of

$$
C(D \times \Omega)=\left\{u: u(., t) \in C_{0}^{\infty}(D) \text { for every } t \in \Omega\right\}
$$

in $W_{\vartheta}^{1, p(. . .)}(D \times \Omega)$. Also, it is obvious that $C(D \times \Omega)$ is a subspace of $W_{0, \vartheta}^{1, p(. .)}(D \times \Omega)$, and the dual space of $W_{0, \vartheta}^{1, p(. .)}(D \times \Omega)$ is $W_{0, \vartheta^{*}}^{-1, q(. .)}(D \times \Omega)$, where $\frac{1}{p(. .)}+\frac{1}{q(. .)}=1$ and $\vartheta^{*}=\vartheta^{1-q(., .)}$.

The following theorem present the Poincaré inequality for the weighted Sobolev spaces $W_{0, \vartheta}^{k, p(.,)}(D \times \Omega)$. For the proof, it can be used the similar method in [16, Theorem 7].

Theorem 2.10. Assume that $p(.,$.$) satisfies the jump condition (see [7]) in D \times \Omega$. Then there is a $C>0$ such that the inequality

$$
\|u\|_{p(., .), \vartheta} \leq C\|\nabla u\|_{p(., .), \vartheta}
$$


holds for every $u \in W_{0, \vartheta}^{k, p(. .)}(D \times \Omega)$ (or $\left.u \in C(D \times \Omega)\right)$.

Therefore, the space $W_{0, \vartheta}^{1, p(. .)}(D \times \Omega)$ equipped with the norm

$$
\||u|\|_{W_{0, \vartheta}^{1, p(. .)}(D \times \Omega)}=\|\nabla u\|_{p(., .), \vartheta}
$$

for $u \in W_{0, \vartheta}^{1, p(. .)}(D \times \Omega)$. It is note that the norms $\|\cdot\|_{W_{\vartheta}^{1, p(., .)}(D \times \Omega)}$ and $\||\cdot|\|_{W_{0, \vartheta}^{1, p(. .)}(D \times \Omega)}$ are equivalent on $W_{\vartheta}^{1, p(. .)}(D \times \Omega)$. Then, $W_{\vartheta}^{1, p(. .)}(D \times \Omega)$ is continuously embedded in $L_{\vartheta}^{p(. .)}(D \times \Omega)$ if and only if the inequality (2.5) is satisfied for every $u \in W_{0, \vartheta}^{1, p(., .)}(D \times \Omega)$.

\section{Compact embedding theorems}

In this section, we present several compact embeddings between the weighted stochastic field variable Lebesgue and Sobolev spaces. Because, we need these embeddings to investigate weak solutions of stochastic partial differential equation (1.1). Now, we will introduce the function $p^{*}(.,$.$) and p_{s}(.,$.$) defined by$

$$
\begin{gathered}
p^{*}(x, t)=\left\{\begin{array}{cc}
\frac{d p(x, t)}{d-p(x, t)} & \text { if } p(x, t)<d, \\
\infty & \text { if } p(x, t) \geq d,
\end{array},\right. \\
p_{s}(x, t)=\frac{p(x, t) s(x, t)}{s(x, t)+1}<p(x, t),
\end{gathered}
$$

and we have

$$
p_{s}^{*}(x, t)=\left\{\begin{array}{cc}
\frac{d p_{s}(x, t)}{d(s(x, t)+1)-p(x, t) s(x, t)} & \text { if } p_{s}(x, t)<d, \\
\operatorname{arbitrary} & \text { if } p_{s}(x, t) \geq d,
\end{array}\right.
$$

for almost all $(x, t) \in D \times \Omega$.

If we use the similar method in [11, Proposition 2.5], then we have the following result.

Proposition 3.1. Assume that the boundary of $D \times \Omega$ possesses the cone property and $p(.,.) \in C(\overline{D \times \Omega})$. Moreover, let $q(.,.) \in C(\overline{D \times \Omega})$ and $1 \leq q(x, t) \leq p^{*}(x, t)$ for $(x, t) \in \overline{D \times \Omega}$. Then the space $W^{1, p(. .)}(D \times \Omega)$ is compactly embedded in $L^{q(. .)}(D \times \Omega)$.

Theorem 3.2. Assume that the boundary of $D \times \Omega$ possesses the cone property, $p(.,.) \in$ $C(\overline{D \times \Omega})$ and $1<p(x, t)$ for all $(x, t) \in \overline{D \times \Omega}$. Suppose that

(i) $0<\vartheta(x, t) \in L^{\alpha(. . .)}(D \times \Omega)$ with $(x, t) \in D \times \Omega, \alpha(.,.) \in C(\overline{D \times \Omega})$ and $1<\alpha^{-}$.

(ii) $1<q(x, t)<\frac{\alpha(x, t)-1}{\alpha(x, t)} p^{*}(x, t)$ for all $(x, t) \in \overline{D \times \Omega}$.

Then, there is a compact embedding from $W^{1, p(. . .)}(D \times \Omega)$ to $L_{\vartheta}^{q(. .)}(D \times \Omega)$.

Proof. For the proof, we use similar method in [6, Theorem 2.1]. Assume that $u \in W^{1, p(., .)}(D \times \Omega)$ and set $r(x, t)=\frac{\alpha(x, t)}{\alpha(x, t)-1} q(x, t)=\alpha_{0}(x, t) q(x, t)$. Then (ii) implies $r(x, t)<p^{*}(x, t)$. This follows that $W^{1, p(., .)}(D \times \Omega) \hookrightarrow \hookrightarrow L^{r(., .)}(D \times \Omega)$ by Proposition 3.1. Moreover, for $u \in W^{1, p(. .)}(D \times \Omega)$, we get $|u|^{q(x, t)} \in L^{\alpha_{0}(x, t)}(D \times \Omega)$. By the Hölder inequality

$$
E\left(\int_{D} \vartheta(x, t)|u|^{q(x, t)} d x\right) \leq\left(1+\frac{1}{p^{-}}-\frac{1}{p^{+}}\right)\|\vartheta\|_{\alpha(., .)}\left\||u|^{q(., .)}\right\|_{\alpha_{0}(., .)}<\infty .
$$

Therefore, we have $W^{1, p(. .)}(D \times \Omega) \subset L_{\vartheta}^{q(. .)}(D \times \Omega)$. Now, assume that $\left(u_{n}\right)_{n \in \mathbb{N}} \subset$ $W^{1, p(.,)}(D \times \Omega)$ and $u_{n} \rightarrow 0$ in $W^{1, p(. .)}(D \times \Omega)$. Since $W^{1, p(., .)}(D \times \Omega)$ is compactly 
embedded in $L^{r(., .)}(D \times \Omega)$ by the Proposition 3.1, we get $u_{n} \longrightarrow 0$ in $L^{r(. .)}(D \times \Omega)$, that is, $\left\|u_{n}\right\|_{r(.,)} \longrightarrow 0$. This yields $\rho_{r(., .)}\left(u_{n}\right) \longrightarrow 0$ and

$$
E\left(\int_{D}\left|u_{n}(x, t)\right|^{r(x, t)} d x\right)=\rho_{\alpha_{0}(. . .)}\left(\left|u_{n}(x, t)\right|^{q(x, t)}\right) \longrightarrow 0
$$

or equivalently

$$
\left\|\left|u_{n}\right|^{q(. .)}\right\|_{\alpha_{0}(., .)} \longrightarrow 0
$$

Thus, we have

$$
E\left(\int_{D} \vartheta(x, t)\left|u_{n}\right|^{q(x, t)} d x\right) \leq\left(1+\frac{1}{p^{-}}-\frac{1}{p^{+}}\right)\|\vartheta\|_{\alpha(.,)}\left\|\left|u_{n}\right|^{q(. .)}\right\|_{\alpha_{0}(. .)} \longrightarrow 0
$$

which implies $\left\|u_{n}\right\|_{q(., .), \vartheta} \longrightarrow 0$. That is the desired result.

Theorem 3.3. Let all conditions in Proposition 3.1 be hold. Moreover, assume that the assumptions in Theorem 3.2 replacing $p(.,$.$) by p_{s}(.,$.$) are also satisfied. Thus we obtain$

$$
W_{\vartheta}^{1, p(., .)}(D \times \Omega) \hookrightarrow \hookrightarrow L_{\vartheta}^{r(. .)}(D \times \Omega),
$$

where $r(x, t) \leq p_{s}^{*}(x, t)$ for all $(x, t) \in D \times \Omega$ and $0<C \leq \vartheta$.

Proof. First of all, we will show that $W_{\vartheta}^{1, p(., .)}(D \times \Omega)$ is continuously embedded in $W^{1, p_{s}(., .)}(D \times \Omega)$. Let $u \in W_{\vartheta}^{1, p(. .)}(D \times \Omega)$. Then it is clear that $u,|\nabla u| \in L_{\vartheta}^{p(. .)}(D \times \Omega)$. If we consider the Hölder inequality, Proposition 2.3 and $\vartheta^{-s(. . .)} \in L^{1}(D \times \Omega)$, then we have

$$
\begin{aligned}
E\left(\int_{D}|\nabla u|^{p_{s}(x, t)} d x\right) & =E\left(\int_{D}|\nabla u|^{p_{s}(x, t)} \vartheta^{\frac{p_{s}(x, t)}{p(x, t)}} \vartheta^{-\frac{p_{s}(x, t)}{p(x, t)}} d x\right) \\
& \leq C\left\||\nabla u|^{p_{s}^{(. . .)}} \vartheta^{\frac{p_{s}(. . .)}{p(. .)}}\right\|_{\frac{p(. . .)}{p_{s}(. .)}}\left\|\vartheta^{-\frac{s(. .)}{s(. .)+1}}\right\|_{s(. .,+1} \\
& \leq C\left(\rho_{p(. .,), \vartheta}(|\nabla u|)\right)^{\frac{1}{\gamma_{1}}}\left(\rho_{s(. .,)}\left(\vartheta^{-1}\right)\right)^{\frac{1}{\gamma_{2}}} \\
& \leq C C_{1}\left(\rho_{p(. . .), \vartheta}(|\nabla u|)\right)^{\frac{1}{\gamma_{1}}}
\end{aligned}
$$

where

$$
\gamma_{1}=\left\{\begin{array}{l}
\left(\frac{p}{p_{s}}\right)^{-}, \text {if }\left\||\nabla u|^{p_{s}(., .)} \vartheta^{\frac{p_{s}(. .)}{p(. .)}}\right\|_{\frac{p(. . .)}{p_{s}(., .)}} \geq 1 \\
\left(\frac{p}{p_{s}}\right)^{+}, \text {if }\left\||\nabla u|^{p_{s}(., .)} \vartheta^{\frac{p_{s}(. . .)}{p(. .)}}\right\|_{\frac{p(. . .)}{p_{s}(.,)}} \leq 1
\end{array}\right.
$$

and

$$
\gamma_{2}=\left\{\begin{array}{cc}
s^{-}+1, & \text { if }\left\|\vartheta^{-\frac{s(., .)}{s(., .)+1}}\right\|_{s(., .)+1} \geq 1 \\
s^{+}+1, & \text { if }\left\|\vartheta^{-\frac{s(., .)}{s(.,)+1}}\right\|_{s(., .)+1} \leq 1 .
\end{array}\right.
$$

Hence, we get

$$
\begin{aligned}
\|\nabla u\|_{p_{s}(., .)}^{\gamma_{3}} & \leq E\left(\int_{D}|\nabla u|^{p_{s}(x, t)} d x\right) \leq C C_{1}\left(\rho_{p(., .), \vartheta}(|\nabla u|)\right)^{\frac{1}{\gamma_{1}}} \\
& \leq C C_{1}\|\nabla u\|_{p(., .),}^{\frac{\gamma_{4}}{\gamma_{1}}}
\end{aligned}
$$


where

and

$$
\gamma_{3}= \begin{cases}p_{s}^{-}, & \text {if }\|\nabla u\|_{p_{s}(. .)} \geq 1 \\ p_{s}^{+}, & \text {if }\|\nabla u\|_{p_{s}(. .)} \leq 1\end{cases}
$$

Therefore, we obtain

$$
\gamma_{4}=\left\{\begin{array}{ll}
p^{+}, & \text {if }\|\nabla u\|_{p(. .,),} \geq 1 \\
p^{-}, & \text {if }\|\nabla u\|_{p(., .),} \leq 1
\end{array} .\right.
$$

$$
\|\nabla u\|_{p_{s}(., .)} \leq C^{*}\|\nabla u\|_{p(., .), \vartheta}^{\frac{\gamma_{4}}{\gamma_{1} \gamma_{3}}} .
$$

Since $p_{s}(.,)<.p(.,$.$) , we have L_{\vartheta}^{p(. .)}(D \times \Omega) \hookrightarrow L^{p(. .)}(D \times \Omega) \hookrightarrow L^{p_{s}(. .)}(D \times \Omega)$, see [9, Theorem 2.8]. Then there exists $C^{* *}>0$ such that

$$
\|u\|_{p_{s}(., .)} \leq C^{* *}\|u\|_{p(.,), \vartheta}
$$

for almost everywhere in $D \times \Omega$. By (3.1) and (3.2), we conclude that $W_{\vartheta}^{1, p(., .)}(D \times \Omega) \subset$ $W^{1, p_{s}(., .)}(D \times \Omega)$. If we consider the Banach theorem in [3], we get $W_{\vartheta}^{1, p(., .)}(D \times \Omega) \hookrightarrow$ $W^{1, p_{s}(., .)}(D \times \Omega)$. This follows from Theorem 3.2 that

$$
W^{1, p_{s}(., .)}(D \times \Omega) \hookrightarrow \hookrightarrow L_{\vartheta}^{r(., .)}(D \times \Omega) .
$$

That is the desired result.

Now, we reveal several required assumptions for the equation (1.1). Assume that $A$ : $\mathbb{R}^{d} \times \Omega \times \mathbb{R} \times \mathbb{R}^{d} \longrightarrow \mathbb{R}^{d}$ and $f: \mathbb{R}^{d} \times \Omega \longrightarrow \mathbb{R}$ satisfy the following growth conditions:

$$
\begin{aligned}
& \left(H_{3}\right)|A(x, t, s, \xi)| \leq \beta \vartheta^{\frac{1}{p(x, t)}}\left[k(x, t)+\vartheta^{\frac{1}{q(x, t)}}|\xi|^{p(x, t)-1}\right] \\
& \left(H_{4}\right) \quad(A(x, t, s, \xi)-A(x, t, s, \mu))(\xi-\mu)>0, \xi \neq \mu \\
& \left(H_{5}\right) A(x, t, s, \xi)|\xi| \geq \alpha \vartheta(x, t)|\xi|^{p(x, t)}
\end{aligned}
$$

where $k(x, t)$ is a positive function in $L^{q(. . .)}(D \times \Omega)$ where $\frac{1}{p(. .)}+\frac{1}{q(. .)}=1$ and $\alpha, \beta$ are positive constants.

Let $A_{0}(x, t, s, \xi): \mathbb{R}^{d} \times \Omega \times \mathbb{R} \times \mathbb{R}^{d} \longrightarrow \mathbb{R}$ be a Carathéodory function such that for a.e. $(x, t) \in \mathbb{R}^{d} \times \Omega$ and for every $s \in \mathbb{R}, \xi \in \mathbb{R}^{d}$, the growth condition

$$
\left|A_{0}(x, t, s, \xi)\right| \leq \gamma(x, t)+g(s) \vartheta(x, t)|\xi|^{p(x, t)-1}
$$

holds where $g: \mathbb{R} \longrightarrow \mathbb{R}^{+}$is a continuous function that belongs to $L^{1}(\mathbb{R})$ and $\gamma(x, t)$ belongs to $L_{\vartheta^{*}}^{q(. .)}(D \times \Omega)$. Finally, we assume that $f \in W_{\vartheta^{*}}^{-1, q(., .)}(D \times \Omega)$.

Lemma 3.4 (see [10]). Assume that $\left(H_{3}\right)-\left(H_{5}\right)$ hold and suppose that $\left\{u_{n}\right\}_{n \in \mathbb{N}}$ is a sequence in $W_{0, \vartheta}^{1, p(. .)}(D \times \Omega)$ such that $u_{n} \rightarrow u$ in $W_{0, \vartheta}^{1, p(. .)}(D \times \Omega)$ and

$$
E\left(\int_{D}\left[A\left(x, t, u_{n}, \nabla u_{n}\right)-A\left(x, t, u_{n}, \nabla u\right)\right] \nabla\left(u_{n}-u\right) d x\right) \longrightarrow 0 .
$$

Then $u_{n} \longrightarrow u$ in $W_{0, \vartheta}^{1, p(., .)}(D \times \Omega)$.

\section{Existence of weak solution of stochastic partial differential equations with stochastic field growth}

Definition 4.1. A function $u \in W_{0, \vartheta}^{1, p(., .)}(D \times \Omega)$ is said to be a weak solution (1.1), if

$$
E\left(\int_{D}\left[A(x, t, u, \nabla u) \nabla \varphi+A_{0}(x, t, u, \nabla u) \varphi\right] d x\right)=E\left(\int_{D} f(x, t) \varphi d x\right)
$$

for all $\varphi \in W_{0, \vartheta}^{1, p(. . .)}(D \times \Omega)$. 
Definition 4.2. A bounded operator $T$ from $W_{0, \vartheta}^{1, p(. .)}(D \times \Omega)$ to its dual $W_{\vartheta^{*}}^{-1, q(., .)}(D \times \Omega)$ is called pseudo-monotone if for any sequences $\left(u_{k}\right)_{k \in \mathbb{N}}$ in $W_{0, \vartheta}^{1, p(. .)}(D \times \Omega)$ satisfying

(i) $u_{k} \rightarrow u$ in $W_{0, \vartheta}^{1, p(. .)}(D \times \Omega)$ as $k \longrightarrow \infty$,

(ii) $\limsup _{k \longrightarrow \infty}\left\langle T\left(u_{k}\right), u_{k}-u\right\rangle \leq 0$,

$T\left(u_{k}\right) \rightarrow T(u)$ and $\left\langle T\left(u_{k}\right), u_{k}\right\rangle \longrightarrow\langle T(u), u\rangle$.

Definition 4.3. Assume that $X$ is a reflexive Banach space and $X^{*}$ denotes dual of $X$. Also, let $\langle.,$.$\rangle be a pair between X$ and $X^{*}$. Then a mapping $\Gamma: X \longrightarrow X^{*}$ is called coercive if there exists a $u \in X$ such that

$$
\frac{\langle\Gamma(u), u\rangle}{\|u\|_{X}} \longrightarrow \infty \text { as }\|u\|_{X} \longrightarrow \infty
$$

Let us define the operator $\Gamma: W_{0, \vartheta}^{1, p(. .)}(D \times \Omega) \longrightarrow W_{\vartheta *}^{-1, q(. . .)}(D \times \Omega)$ by

$$
\langle\Gamma(u), \varphi\rangle=E\left(\int_{D}\left[A(x, t, u, \nabla u) \nabla \varphi+A_{0}(x, t, u, \nabla u) \varphi\right] d x\right)
$$

for all $\varphi \in W_{0, \vartheta}^{1, p(. .)}(D \times \Omega)$ and $\frac{1}{p(. .)}+\frac{1}{q(. .)}=1$. Hence, we can write the equation (1.1) as $\langle\Gamma(u), \varphi\rangle=\langle f, \varphi\rangle$.

Proposition 4.4 (Weak compactness of bounded set, see [12]). Suppose that $X$ is a reflexive Banach space. Moreover, assume that $\left(u_{k}\right)_{k \in \mathbb{N}}$ is a sequence such that

(i) $u_{k} \in X$

(ii) $\left\|u_{k}\right\|_{X} \leq C$ for all $k \in \mathbb{N}$,

that is, $\left(u_{k}\right)_{k \in \mathbb{N}}$ is a bounded sequence in $X$, then there exists a subsequence $\left(u_{k_{l}}\right)_{l \in \mathbb{N}}$ and an element $u_{0} \in X$ such that $u_{k_{l}} \rightarrow u_{0}$ in $X$.

Theorem 4.5 (see [12]). Let $X$ be a reflexive Banach space and assume that $\Gamma: X \longrightarrow X^{*}$ is continuous (bounded), coercive and pseudo-monotone. Then for every $g \in X^{*}$ there exists a solution $u \in X$ of the equation $\Gamma(u)=g$.

Now, we are ready to give our main motivation of the paper.

Theorem 4.6. If the conditions $\left(H_{1}\right)-\left(H_{5}\right)$ hold, then there exists at least a weak solution of (1.1) in $W_{0, \vartheta}^{1, p(. .)}(D \times \Omega)$.

Proof. The proof is based on three parts.

Step 1. First of all, we will show that the operator $\Gamma$ is bounded. The operator $\Gamma$ is equal to the sum of two operators such that $\Gamma=\Gamma_{1}+\Gamma_{2}$ where

$$
\left\langle\Gamma_{1}(u), \varphi\right\rangle=E\left(\int_{D} A(x, t, u, \nabla u) \nabla \varphi d x\right)
$$

and

$$
\left\langle\Gamma_{2}(u), \varphi\right\rangle=E\left(\int_{D} A_{0}(x, t, u, \nabla u) \varphi d x\right) .
$$


If we consider $\left(H_{3}\right)$, Proposition 2.3 and Hölder inequality, then we have

$$
\begin{aligned}
\left|\left\langle\Gamma_{1}(u), \varphi\right\rangle\right| \leq & C\left\|A(x, t, u, \nabla u) \vartheta^{-\frac{1}{p(x, t)}}\right\|_{q(. .)}\left\|\nabla \varphi \vartheta^{\frac{1}{p(x, t)}}\right\|_{p(., .)} \\
= & C\left\|A(x, t, u, \nabla u) \vartheta^{-\frac{1}{p(x, t)}}\right\|_{q(. .)}\||\varphi|\|_{W_{0, \vartheta}^{1, p(.,)}(D \times \Omega)} \\
\leq & C\left[E\left(\int|A(x, t, u, \nabla u)|^{q(x, t)} \vartheta^{-\frac{q(x, t)}{p(x, t)}} d x\right)\right]^{\theta}\||\varphi|\|_{W_{0, \vartheta}^{1, p(. .)}(D \times \Omega)} \\
\leq & C\left[E \left(\int_{D} \mid \beta \vartheta^{\frac{1}{p(x, t)}}[k(x, t)\right.\right. \\
& \left.\left.\left.+\vartheta^{\frac{1}{q(x, t)}}|\nabla u|^{p(x, t)-1}\right]\left.\right|^{q(x, t)} \vartheta^{-\frac{q(x, t)}{p(x, t)}} d x\right)\right]^{\theta}\||\varphi|\|_{W_{0, \vartheta}^{1, p(. .)}(D \times \Omega)} \\
\leq & C^{*}\left(\max \left\{\beta^{q^{-}}, \beta^{q^{+}}\right\}\right)^{\theta}\left[\int _ { D } \left[|k(x, t)|^{q(x, t)}\right.\right. \\
& \left.\left.\left.+|\nabla u|^{p(x, t)} \vartheta\right] d x\right)\right]^{\theta}\|\varphi \mid\|_{W_{0, \vartheta}^{1, p(. .)}(D \times \Omega)} \\
\leq & C^{*}\left(C_{1}+\rho_{p(. .,), \vartheta}(\nabla u)\right)^{\theta}\|\mid\|_{W_{0, \vartheta}^{1, p(. .)}(D \times \Omega)}
\end{aligned}
$$

where

$$
\theta=\left\{\begin{array}{l}
\frac{1}{q^{-}}, \text {if }\|A(x, t, u, \nabla u)\|_{q(. .,), \vartheta^{*}} \geq 1 \\
\frac{1}{q^{+}}, \text {if }\|A(x, t, u, \nabla u)\|_{q(. .,), \vartheta^{*}} \leq 1
\end{array},\right.
$$

and $\frac{1}{p(. . .)}+\frac{1}{q(. .)}=1$. This yields that $\Gamma_{1}$ is bounded. In a similar way, since $\gamma \in$ $L_{\vartheta *}^{q(. .)}(D \times \Omega)$, we get

$$
\begin{aligned}
& \left|\left\langle\Gamma_{2}(u), \varphi\right\rangle\right| \leq C\left\|A_{0}(x, t, u, \nabla u) \vartheta^{-\frac{1}{p(x, t)}}\right\|_{q(. . .)}\left\|\varphi \vartheta^{\frac{1}{p(x, t)}}\right\|_{p(. . .)} \\
& \leq C\left\|A_{0}(x, t, u, \nabla u) \vartheta^{-\frac{1}{p(x, t)}}\right\|_{q(. . .)}\||\varphi|\|_{W_{0, \vartheta}^{1, p(. . .)}(D \times \Omega)} \\
& \leq C\left[E\left(\int_{D}\left|A_{0}(x, t, u, \nabla u)\right|^{q(x, t)} \vartheta^{-\frac{q(x, t)}{p(x, t)}} d x\right)\right]^{\eta}\||\varphi|\|_{W_{0, \vartheta}^{1, p(., .)}(D \times \Omega)} \\
& \leq C\left[E\left(\left.\left.\int_{D}|\gamma(x, t)+g(u) \vartheta| \nabla u\right|^{p(x, t)-1}\right|^{q(x, t)} \vartheta^{-\frac{q(x, t)}{p(x, t)}} d x\right)\right]^{\eta}\||\varphi|\|_{W_{0, \vartheta}^{1, p(. .)}(D \times \Omega)} \\
& \leq C^{*}\left[E\left(\int_{D}|\gamma(x, t)|^{q(x, t)} \vartheta^{-\frac{q(x, t)}{p(x, t)}} d x\right)\right. \\
& \left.+E\left(\int_{D}|g(u)|^{q(x, t)}|\nabla u|^{p(x, t)} \vartheta d x\right)\right]^{\eta}\||\varphi|\|_{W_{0, \vartheta}^{1, p(., .)}(D \times \Omega)} \\
& \leq C^{*}\left[\rho_{q(., .), \vartheta^{*}}(\gamma)+\max \left\{\|g\|_{\infty}^{q^{-}},\|g\|_{\infty}^{q^{+}}\right\} \rho_{p(., .), \vartheta}(\nabla u)\right]^{\eta}\||\varphi|\|_{W_{0, \vartheta}^{1, p(., .)}(D \times \Omega)}
\end{aligned}
$$


where

$$
\eta=\left\{\begin{array}{l}
\frac{1}{q^{-}}, \text {if }\left\|A_{0}(x, t, u, \nabla u)\right\|_{q(., .), \vartheta^{*}} \geq 1 \\
\frac{1}{q^{+}}, \text {if }\left\|A_{0}(x, t, u, \nabla u)\right\|_{q(. .), \vartheta^{*}} \leq 1
\end{array},\right.
$$

and $\frac{1}{p(., .)}+\frac{1}{q(\cdot, .)}=1$. Thus, we obtain that $\Gamma_{2}$ is bounded. Therefore, we get that $\Gamma$ is bounded.

Step 2. Now, we will show that the operator $\Gamma$ is coercive. By $\left(H_{5}\right)$, we have

$$
\begin{aligned}
\frac{\left\langle\Gamma_{1}(u), u\right\rangle}{\||u|\|_{W_{0, \vartheta}^{1, p(.,)}(D \times \Omega)}} & \geq \frac{E\left(\int_{D} \alpha|\nabla u|^{p(x, t)} \vartheta(x, t) d x\right)}{\|\nabla u\|_{p(., .), \vartheta}} \\
& =\frac{\alpha \rho_{p(., .), \vartheta}(\nabla u)}{\|\nabla u\|_{p(., .), \vartheta}} \geq C\|\nabla u\|_{p(., .), \vartheta}^{r}
\end{aligned}
$$

for some $r>1$. On the other hand, since the norm $\left\|A_{0}(x, t, u, \nabla u)\right\|_{q(., .), \vartheta^{*}}$ is bounded, then we have

$$
\begin{aligned}
\left|\left\langle\Gamma_{2}(u), u\right\rangle\right| & \leq C\left\|A_{0}(x, t, u, \nabla u)\right\|_{q(., .), \vartheta^{*}}\|u\|_{p(., .), \vartheta} \\
& \leq C^{*}\||u|\|_{W_{0, \vartheta}^{1, p(.,)}(D \times \Omega)}
\end{aligned}
$$

This follows that

$$
\frac{\langle\Gamma(u), u\rangle}{\||u|\|_{W_{0, \vartheta}^{1, p(., .)}(D \times \Omega)}} \longrightarrow \infty \text { as }\|\mid u\|_{W_{0, \vartheta}^{1, p(., .)}(D \times \Omega)} \longrightarrow \infty .
$$

Step 3. Now, we will obtain that the operator $\Gamma$ is pseudo-monotone from $W_{0, \vartheta}^{1, p(. .)}(D \times \Omega)$ to $W_{\vartheta^{*}}^{-1, q(., .)}(D \times \Omega)$. Let $u_{k} \rightarrow u$ in $W_{0, \vartheta}^{1, p(.,)}(D \times \Omega)$ and $\limsup _{k \longrightarrow \infty}\left\langle\Gamma\left(u_{k}\right), u_{k}-u\right\rangle \leq 0$. Since $\Gamma$ is bounded and $u_{k} \rightarrow u$, then we have

$$
\Gamma\left(u_{k}\right) \rightarrow h \text { in } W_{\vartheta^{*}}^{-1, q(. .)}(D \times \Omega) .
$$

By (4.1), we can write that

$$
\limsup _{k \longrightarrow \infty}\left\langle\Gamma\left(u_{k}\right), u_{k}\right\rangle \leq\langle h, u\rangle
$$

By the growth condition $\left(H_{3}\right)$ and Proposition 4.4 , the sequence $\left(A\left(x, t, u_{k}, \nabla u_{k}\right)\right)_{k \in \mathbb{N}}$ is bounded in $\left(L_{\vartheta^{*}}^{q(.,)}(D \times \Omega)\right)^{d}$ such that

$$
A\left(x, t, u_{k}, \nabla u_{k}\right) \rightarrow \varphi \text { in }\left(L_{\vartheta^{*}}^{q(. .)}(D \times \Omega)\right)^{d}
$$

as $k \longrightarrow \infty$. Similarly, since $\left(A_{0}\left(x, t, u_{k}, \nabla u_{k}\right)\right)_{k}$ is bounded in $L_{\vartheta^{*}}^{q(. .)}(D \times \Omega)$, then there exists a function $\psi \in L_{\vartheta^{*}}^{q(. .)}(D \times \Omega)$ such that

$$
A_{0}\left(x, t, u_{k}, \nabla u_{k}\right) \rightarrow \psi \text { in } L_{\vartheta^{*}}^{q(. .)}(D \times \Omega)
$$


as $k \longrightarrow \infty$. For all $v \in W_{0, \vartheta}^{1, p(. .)}(D \times \Omega)$, we have

$$
\begin{aligned}
\langle h, v\rangle= & \lim _{k \longrightarrow \infty}\left\langle\Gamma\left(u_{k}\right), v\right\rangle \\
= & \lim _{k \longrightarrow \infty} E\left(\int_{D} A\left(x, t, u_{k}, \nabla u_{k}\right) \nabla v d x\right) \\
& +\lim _{k \longrightarrow \infty} E\left(\int_{D} A_{0}\left(x, t, u_{k}, \nabla u_{k}\right) v d x\right) \\
= & E\left(\int_{D} \varphi \nabla v d x\right)+E\left(\int_{D} \psi v d x\right) .
\end{aligned}
$$

Since $W_{0, \vartheta}^{1, p(. .)}(D \times \Omega)$ is compactly embedded in $L_{\vartheta}^{p(. .)}(D \times \Omega)$ by Theorem 3.3 , we get

$$
u_{k} \longrightarrow u \text { in } L_{\vartheta}^{p(. .)}(D \times \Omega) \text { and a.e. in } D \times \Omega \text {. }
$$

By (4.4) and (4.6), we have

$$
E\left(\int_{D} A_{0}\left(x, t, u_{k}, \nabla u_{k}\right) u_{k} d x\right) \rightarrow E\left(\int_{D} \psi u d x\right)
$$

as $k \longrightarrow \infty$. On the other hand, if we consider (4.2) and (4.5), then we have

$$
\begin{aligned}
& \limsup _{k \longrightarrow \infty}\left\langle\Gamma\left(u_{k}\right), u_{k}\right\rangle \\
& \quad=\limsup _{k \longrightarrow \infty}\left(E\left(\int_{D} A\left(x, t, u_{k}, \nabla u_{k}\right) \nabla u_{k} d x\right)+E\left(\int_{D} A_{0}\left(x, t, u_{k}, \nabla u_{k}\right) u_{k} d x\right)\right) \\
& \quad \leq E\left(\int_{D} \varphi \nabla u d x\right)+E\left(\int_{D} \psi u d x\right) .
\end{aligned}
$$

Hence we obtain

$$
\limsup _{k \rightarrow \infty} E\left(\int_{D} A\left(x, t, u_{k}, \nabla u_{k}\right) \nabla u_{k} d x\right) \leq E\left(\int_{D} \varphi \nabla u d x\right) .
$$

Due to $\left(H_{4}\right)$, we have

$$
E\left(\int_{D}\left(A\left(x, t, u_{k}, \nabla u_{k}\right)-A\left(x, t, u_{k}, \nabla u\right)\right)\left(\nabla u_{k}-\nabla u\right) d x\right)>0
$$

and

$$
\begin{aligned}
& E\left(\int_{D} A\left(x, t, u_{k}, \nabla u_{k}\right) \nabla u_{k} d x\right) \\
& \geq-E\left(\int_{D} A\left(x, t, u_{k}, \nabla u\right) \nabla u d x\right)+E\left(\int_{D} A\left(x, t, u_{k}, \nabla u_{k}\right) \nabla u d x\right) \\
& \quad+E\left(\int_{D} A\left(x, t, u_{k}, \nabla u\right) \nabla u_{k} d x\right) .
\end{aligned}
$$


Moreover, by (4.3), we get

$$
\liminf _{k \rightarrow \infty} E\left(\int_{D} A\left(x, t, u_{k}, \nabla u_{k}\right) \nabla u_{k} d x\right) \geq E\left(\int_{D} \varphi \nabla u d x\right) .
$$

It is obtained that

$$
\lim _{k \longrightarrow \infty} E\left(\int_{D} A\left(x, t, u_{k}, \nabla u_{k}\right) \nabla u_{k} d x\right)=E\left(\int_{D} \varphi \nabla u d x\right)
$$

by (4.8). If we consider (4.5), (4.7) and (4.9), then we have

$$
\lim _{k \longrightarrow \infty}\left\langle\Gamma\left(u_{k}\right), u_{k}\right\rangle=\langle h, u\rangle .
$$

This follows from $(4.6)$ and $\left(H_{3}\right)$ that

$$
A\left(x, t, u_{k}, \nabla u\right) \longrightarrow A(x, t, u, \nabla u)
$$

in $\left(L_{\vartheta^{*}}^{q(. .)}(D \times \Omega)\right)^{d}$. Hence, we get

$$
\lim _{k \longrightarrow \infty} E\left(\int_{D}\left(A\left(x, t, u_{k}, \nabla u_{k}\right)-A\left(x, t, u_{k}, \nabla u\right)\right)\left(\nabla u_{k}-\nabla u\right) d x\right)=0 .
$$

By Lemma 3.4, we obtain

$$
u_{k} \longrightarrow u \text { in } W_{0, \vartheta}^{1, p(. .)}(D \times \Omega)
$$

and then $\nabla u_{k} \longrightarrow \nabla u$ a.e. in $D \times \Omega$ for a subsequence denoted by $\left(u_{k}\right)_{k \in \mathbb{N}}$. Since $A$ and $A_{0}$ are Carathéodory functions, we have

$$
\begin{aligned}
A\left(x, t, u_{k}, \nabla u_{k}\right) & \longrightarrow A\left(x, t, u_{k}, \nabla u\right) \\
A_{0}\left(x, t, u_{k}, \nabla u_{k}\right) & \longrightarrow A_{0}\left(x, t, u_{k}, \nabla u\right) .
\end{aligned}
$$

This yields that $h=\Gamma(u)$ and the operator $\Gamma$ is pseudo-monotone. Finally, if we consider the Theorem 4.5, then there exists at least a weak solution of (1.1).

\section{References}

[1] H. Aoyama, Lebesgue spaces with variable on a probability space, Hiroshima Math. J. 39, 207-216, 2009.

[2] F.E. Browder, Pseudo-monotone operators and nonlinear elliptic boundary value problems on unbounded domains, Proc. Natl. Acad. Sci. USA, 74 (7), 2659-2661, 1977.

[3] H. Cartan, Differential Calculus, Herman, Paris-France, 1971.

[4] B. Cekic, R. Mashiyev and G.T. Alisoy, On the Sobolev-type inequality for Lebesgue spaces with a variable exponent, Int. Math. Forum, 1 (27), 1313-1323, 2006.

[5] L. Diening, P. Harjulehto, P. Hästö and M. Růžička, Lebesgue and Sobolev Spaces with Variable Exponents, Springer-Verlag, Berlin, 2011.

[6] X.L. Fan, Solutions for $p(x)$-Laplacian Dirichlet problems with singular coefficients, J. Math. Anal. Appl. 312, 464-477, 2005.

[7] P. Harjulehto, P. Hästö, M. Koskenoja and S. Varonen, The Dirichlet energy integral and variable exponent Sobolev spaces with zero boundary values, Potential Anal. 25, 205-222, 2006.

[8] P. Harjulehto, P. Hästö, U.V. Le and M. Nuortio, Overview of differential equations with non-standard growth, Nonlinear Anal. 72, 4551-4574, 2010.

[9] O. Kováčik and J. Rákosník, On spaces $L^{p(x)}$ and $W^{k, p(x)}$, Czechoslovak Math. J. 41(116) (4), 592-618, 1991.

[10] B. Lahmi, E. Azroul and K. El Haitin, Nonlinear degenerated elliptic problems with dual data and nonstandard growth, Math. Reports 20(70) (1), 81-91, 2018. 
[11] R.A. Mashiyev, S. Oğraş, Z. Yucedag and M. Avci, The Nehari manifold approach for Dirichlet problem involving the $p(x)$-Laplacian equation, J. Korean Math. Soc. 47 (4), 845-860, 2010.

[12] M. Renardy and R.C. Rogers, An Introduction to Partial Differential Equations (Second Edition), Springer, 2004.

[13] M. Růžička, Electrorheological fluids: modeling and mathematical theory, Springer, Berlin Heidelberg New York, 2000.

[14] B. Tian, Y. Fu and B. Xu, Function spaces with a random variable exponent, Abstr. Appl. Anal. 2011, Article ID 179068, 2011.

[15] B. Tian, B. Xu and Y. Fu, Stochastic field exponent function spaces with applications, Complex Var. Elliptic Equ. 59 (1), 133-148, 2014.

[16] C. Unal and I. Aydın, Weighted variable exponent Sobolev spaces with zero boundary values and capacity estimates, Sigma J. Eng. \& Nat. Sci. 36 (2), 371-386, 2018. 